\title{
CHANGING TIME HISTORY IN MOVING BOUNDARY PROBLEMS*
}

\author{
DONALD S. COHEN $\dagger$ AND THOMAS ERNEUX $\ddagger$
}

\begin{abstract}
This paper is dedicated to Edward L. Reiss on the occasion of his 60 th birthday.
\end{abstract}
\begin{abstract}
A class of diffusion-stress equations modeling transport of solvent in glassy polymers is considered. The problem is formulated as a one-phase Stefan problem. It is shown that the moving front changes like $\sqrt{t}$ initially but quickly behaves like $t$ as $t$ increases. The $t$ behavior is typical of stress-dominated transport. The quasi-steady state approximation is used to analyze the time history of the moving front. This analysis is motivated by the small time solution.
\end{abstract}

Key words. one phase Stefan problem, diffusion in polymers

1. Introduction. There does not yet exist a satisfactory theory or explanation for transport in glassy polymers. Since glassy polymers are of enormous technological importance, there is strong motivation to develop a comprehensive and useful theory. With regard to polymer-penetrant systems, of particular interest is the time history of a diffusing front of a penetrant through the polymers.

According to classical diffusion theory, the position $x=S(t)$ of a moving boundary is given by $x=($ constant $) t^{1 / 2}$ with corresponding speed proportional to $t^{-1 / 2}$. This follows from solving a so-called one-phase Stefan problem for the concentration $C(x, t)$ behind a moving boundary at position $x=S(t)$. We must find both $C(x, t)$ and $S(t)$, where $C$ satisfies the classical Fick's diffusion equation, $C_{t}=\left(D C_{x}\right)_{x}$, subject to various initial and boundary conditions involving $S(t)$. Here $D$ is the diffusivity of the medium and $D$ may even depend on $C$.

Since the work of Alfrey, Gurnee, and Lloyd [1], it has been generally recognized that in polymer-penetrant systems the motion of the penetrant front can proceed according to laws considerably different from the classical simple $t^{1 / 2}$ of Fickian diffusion, and in fact, in a given problem the front may exhibit a fairly general time dependence. We refer to this as a changing time history. Furthermore, there is no one simple alternative to the classical situation. Although some global principles are starting to emerge, the fact is that there is no universal theory, and different models (theories, equations of motion) are needed for systems of one type which may differ considerably from those of other types of systems depending on type of polymer, type of diffusing substance, and ambient conditions.

When the front moves with near constant speed (and thus with position proportional to $t$ ), the behavior is called Case II diffusion to distinguish it from Case I (or Fickian) diffusion. As shown by Cox and Cohen [2], one explanation for Case II diffusion (as well as many other time histories) is that the Fickian flux must be supplemented by a flux due to pressure gradients. Appropriate equations of motion

* Received by the editors June 12, 1989; accepted for publication (in revised form) July 25, 1989.

$\dagger$ Department of Applied Mathematics, California Institute of Technology, Pasadena, California 91125. This research was supported in part by the United States Army Research Office (Durham), Contract DAAL03-89-K-0014, National Science Foundation grant DMS-88706642, and Air Force Office of Scientific Research grant AFOSR-88-0269.

$\ddagger$ Department of Engineering Sciences and Applied Mathematics, Northwestern University, Evanston, Illinois 60208. This research was supported in part by National Science Foundation grant DMS-8701302 and United States Air Force Office of Scientific Research grant AFOSR 85-0150. 
for the polymer-penetrant system are:

$$
\begin{gathered}
C_{t}=\left(D C_{x}+E \sigma_{x}\right)_{x}, \\
\sigma_{t}+\beta \sigma=\mu C .
\end{gathered}
$$

Here $C$ is the concentration of the diffusing penetrant, and $\sigma$ is the stress induced. $D, E, \beta$, and $\mu$ may all depend on $C$ and/or $\sigma$; their properties are discussed further in [2] and [3] based on the experimental results of Thomas and Windle [4]-[5]. Problems for a penetrant front involving these equations are formulated as moving boundary problems. A moving boundary located at $x=L(t)$ separates a region of the polymer where $C \neq 0$ and a region of the polymer where $C=0$. Because the stress interferes with the normal diffusive transport of the solvent, this moving boundary varies with different rates as $t \rightarrow 0$ or as $t \rightarrow \infty$. These moving boundary problems are mathematically particularly interesting and difficult because the changing time history precludes the use of a similarity variable which is commonly emphasized in traditional Stefan problems to facilitate their solution. In this paper, we shall account for observations in which the solvent front velocity, initially proportional to $t^{-1 / 2}$, changes and becomes nearly constant for a long period of time. Of practical interest is the prediction of this changing time history. The establishing and maintaining of a constant front velocity is of crucial importance because constant flux rates are desirable in many modern technological procedures [6], [7]. In [7], we propose a specific model for the controlled release of pharmaceuticals which use (1.1) and (1.2).

In $\S 2$ we formulate the prototype moving-boundary problem involving (1.1) and (1.2) to account for Case II diffusion and the transition to it. We take the so-called stress-driven regime [2] which introduces a small parameter multiplying $D$ in the dimensionless equations. Realistic scalings to achieve this are given in [2]. An asymptotic method valid for short times is employed in $\S 3$. We show that for very short times the process is diffusion dominated so that the front initially penetrates on $t^{1 / 2}$ time scales. In $\S 4$, we consider the quasi-steady state approximation and analyze the complete time history of the moving front.

2. Formulation. We consider a glassy polymer of finite length $0 \leqq x \leqq x_{0}$ exposed at $x=0$ to a reservoir of solvent at fixed concentration $C=1$. In contact with the solvent, the polymer swells and interferes with the diffusive transport of the solvent. The solvent front velocity, initially proportional to $t^{-1 / 2}$, changes and becomes almost constant. As shown in [2], [3] this response can be modeled by employing equations of the form (1.1) and (1.2). They describe the interaction of diffusion and stress through a viscoelastic response where the stress depends upon the amount of penetrant present. Assuming for simplicity a linear viscoelastic polymer and a simple linear relation between the total flux of solvent and the gradients of $C$ and $\sigma$, the free boundary problem is then formulated as follows (see Fig. 1): The concentration $C(x, t)$ and the stress $\sigma(x, t)$ satisfy

$$
\begin{gathered}
C_{t}=\varepsilon C_{x x}+\sigma_{x x}, \quad 0<x<L(t), \quad t>0, \\
\sigma_{t}=C-\sigma, \quad 0<x<L(t), \quad t>0 .
\end{gathered}
$$

The boundary conditions at $x=0$ and $x=L(t)$ are given by

$$
C(0, t)=1, \quad t>0,
$$



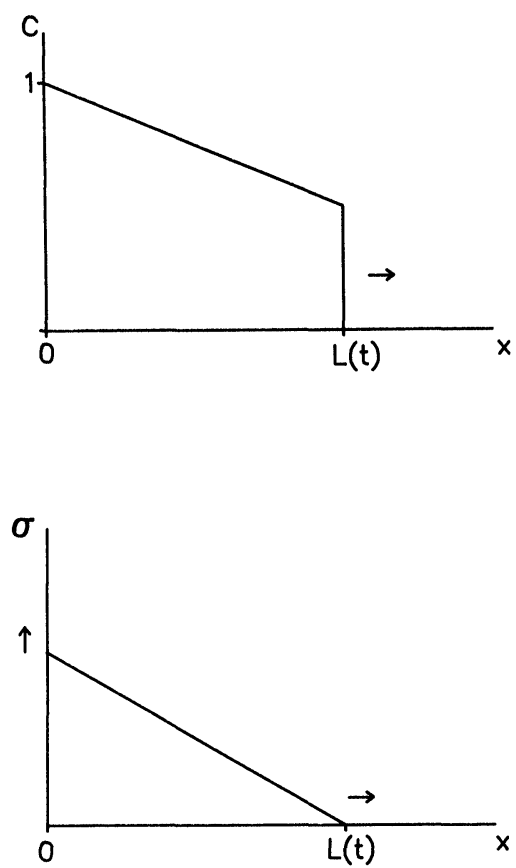

FIG. 1. The one-phase Stefan problem. The concentration $C$ and the stress $\sigma$ are represented as functions of $x$ for $0<x<L(t)$. The moving front is located at $x=L(t)$ and is characterized by discontinuous values of $C$ and $\sigma_{x}$.

$$
\left.\begin{array}{rl}
-\varepsilon C_{x}-\sigma_{x} & =C \frac{d L}{d t}, \\
\sigma & =0, \\
\sigma_{x x} & =0,
\end{array}\right\} \text { at } x=L(t) .
$$

Moreover, we assume the following initial conditions:

$$
\sigma(0,0)=L(0)=0
$$

Note that $C(0,0)=0$, although we have $C(0, t)=1, t>0$. The constant $\varepsilon$ is positive but small compared to 1 . Condition (2.4) results from a mass balance at the moving front assuming that $C=0$ in the dry polymer, and condition (2.5) is clearly suggested since $\sigma=0$ in the dry polymer. The condition (2.6) is one of a reasonable class of conditions suggested by the numerical work of Cox [8] for one class of polymerpenetrant system.

In order to analyze the short time behavior of the solution of (2.1)-(2.7), it is mathematically convenient to solve the moving boundary problem in a fixed interval. To this end, we introduce a new spatial variable defined by

$$
z=\frac{x}{L(t)} \text {. }
$$

In terms of (2.8), our problem (2.1)-(2.7) can be reformulated as

$$
\begin{gathered}
L^{2} C_{t}-L L^{\prime} z C_{z}=\varepsilon C_{z z}+\sigma_{z z}, \quad 0<z<1, \quad t>0, \\
L \sigma_{t}-L^{\prime} z \sigma_{z}=L(C-\sigma), \quad 0<z<1, \quad t>0,
\end{gathered}
$$




$$
\begin{gathered}
C(0, t)=1, \quad t>0, \\
-\varepsilon C_{z}-\sigma_{z}=C L L^{\prime}, \\
\sigma=0, \\
\sigma_{z z}=0, \\
\sigma(z, 0)=0, L(0)=0 .
\end{gathered}
$$

We shall construct various asymptotic expansions of the solution in the next sections. As we shall see, the solution possesses a changing time history. Thus, no similarity variable exists which would enable us to transform our problem to a simple one involving ordinary differential equations. It is primarily in this respect that our problem differs from standard classical moving boundary problems (i.e., Stefan problems) of diffusive type.

3. Short time behavior. For small times we seek a solution of (2.9)-(2.15) in the form

$$
\begin{gathered}
C(z, t)=C_{0}(z)+t C_{1}(z)+\cdots, \\
\sigma(z, t)=t \sigma_{1}(z)+t^{2} \sigma_{2}(z)+\cdots, \\
L(t)=t^{1 / 2}(\alpha+\beta t+\cdots) .
\end{gathered}
$$

The expansion (3.2) for $\sigma(z, t)$ is motivated by the behavior of $\sigma(0, t)$. Indeed from (2.10) evaluated at $z=0$, we find that

$$
\sigma(0, t)=1-e^{-t}
$$

which behaves like $t$ as $t \rightarrow 0$. The $t^{1 / 2}$ scaling of (3.3) is motivated by the idea that diffusion is the dominant mechanism for short time. Introducing (3.1)-(3.3) into (2.9)-(2.15), we find that the leading order equations are given by

$$
\begin{gathered}
\varepsilon C_{0}^{\prime \prime}+\frac{1}{2} \alpha^{2} z C_{0}^{\prime}=0, \\
\frac{1}{2} z \sigma_{1}^{\prime}-\sigma_{1}+C_{0}=0, \\
C_{0}(0)=1, \\
\sigma_{1}(1)=\sigma_{1}^{\prime \prime}(1)=0, \\
\varepsilon C_{0}^{\prime}(1)+\frac{1}{2} \alpha^{2} C_{0}(1)=0 .
\end{gathered}
$$

Thus, we must find the functions $C_{0}(z)$ and $\sigma_{1}(z)$ and the number $\alpha$ satisfying (3.5)-(3.9).

First we find $\alpha$. Upon evaluating (3.6) at $z=1$ and using the fact that $\sigma_{1}(1)=0$ we obtain

$$
\frac{1}{2} \sigma_{1}^{\prime}(1)+C_{0}(1)=0
$$

Next, we differentiate (3.6) and evaluate the resulting expression at $z=1$ using the fact that $\sigma_{1}^{\prime \prime}(1)=0$ to obtain

$$
\frac{1}{2} \sigma_{1}^{\prime}(1)-C_{0}^{\prime}(1)=0 .
$$

Equations (3.9), (3.10), and (3.11) constitute three simultaneous equations in the three unknowns $\sigma(1), C_{0}^{\prime}(1)$, and $\sigma_{1}^{\prime}(1)$. A nontrivial solution will exist only for

$$
\alpha=\sqrt{2 \varepsilon} .
$$

Next, using this value for $\alpha$, we solve equation (3.5) subject to condition (3.7) to find

$$
C_{0}(z)=1+d_{1} \int_{0}^{z} e^{-y^{2} / 2} d y
$$


where $d_{1}$ is a constant. The value for $d_{1}$ is determined by using (3.9) with $\alpha$ given by (3.12). In this way we obtain

$$
C_{0}(z)=1-\frac{\int_{0}^{z} e^{-y^{2} / 2} d y}{e^{-1 / 2}+\int_{0}^{1} e^{-y^{2} / 2} d y},
$$

which can be expressed in terms of error functions if we choose. Finally we find that

$$
\sigma_{1}(z)=2 z^{2} \int_{z}^{1} y^{-3} C_{0}(y) d y .
$$

Therefore, to leading order we find that $L(t) \sim \sqrt{2 \varepsilon t}, C \sim C_{0}(z)$ and $\sigma \sim t \sigma_{1}(z)$ where $C_{0}(z)$ and $\sigma_{1}(z)$ are given by (3.14) and (3.15), respectively.

4. The quasi-steady state approximation. Upon expanding the exponentials in (3.14) in a Taylor's series, we find that

$$
C_{0}(z)=1-\frac{z}{2}\left[1+O\left(\frac{1}{6} z^{2}\right)\right] .
$$

We note that (4.1a) is a numerically good approximation even if $z=1$. We have verified that $(4.1 \mathrm{a})$ can be obtained directly from the original equations by neglecting the $C_{t}$ term in (2.1). In classical moving boundary problems of certain types, a common engineering approximation is the so-called quasi-steady state approximation [9, p. 310], wherein it is assumed that the concentration distribution behind the moving boundary approximates at any instant the steady-state distribution, which would be set up if the boundary were fixed in position at that instant. Analytically, in our problem this amounts to neglecting the $C_{t}$ term in (2.1). More formally we replace the $C_{t}$ term on the left-hand side of $(2.1)$ by $\eta C_{t}$, where $\eta$ is a small parameter, and seek a solution for $C, \sigma$, and $L$ as a power series in $\eta$. The leading terms in the series are then given by the solution of (2.1)-(2.7) with $\eta=0$.

We shall now assume that the quasi-steady approximation remains valid for small and moderate time and use it to determine analytically the history of the moving point. In a different diffusive moving boundary problem, Cohen and Erneux [7] have justified the quasi-steady approximation for all time by showing that it constitutes the leading term in a formal perturbation theory. (More precisely, it is the leading term in the outer expansion in a singular perturbation approach.) We have been unable to carry out the same analysis here. However, its numerical validity for small times as shown above, and a numerical study [8] of equations (2.1) and (2.2), suggest its validity for small and moderate time.

We now study (2.1)-(2.7) with $C_{t}$ on the left-hand side of (2.1) taken to be identically zero. Thus, $(2.1)$ is replaced by $\varepsilon C_{x x}+\sigma_{x x}=0$. Just as in $\S 2$ we introduce $z$ given by (2.8) and we obtain the system

$$
\begin{gathered}
\varepsilon C_{z z}+\sigma_{z z}=0, \quad 0<z<1, \quad t>0, \\
L \sigma_{t}-L^{\prime} z \sigma_{z}=L(C-\sigma), \quad 0<z<1, \quad t>0,
\end{gathered}
$$

subject to conditions (2.10)-(2.15).

Upon integrating (4.1b) twice and using (2.10) and (2.12), we obtain

$$
-\varepsilon(C-1)-(\sigma-\sigma(0, t))=C(1, t) L L^{\prime} z
$$

Here $\sigma(0, t)$ is obtained from (4.2) evaluated at $z=0$ and is given by (3.4). The form of equations (4.2) and (4.3) as well as the boundary condition (2.12) suggest seeking 
solutions for $C$ and $\sigma$ which are linear in $z$. Specifically, we assume that

$$
\begin{gathered}
C=1+A(t) z, \\
\sigma=\sigma(0, t)(1-z) .
\end{gathered}
$$

By substituting (4.4) and (4.5) into (4.2), (4.3) and the boundary conditions (2.11)(2.14), we find

$$
\begin{gathered}
-\varepsilon A+\sigma(0, t)=(1+A) L L^{\prime} \\
-L+L^{\prime} \sigma(0, t)=L A .
\end{gathered}
$$

By eliminating $A$, we obtain the following nonlinear differential equations for the evolution of $L(t)$ :

$$
\begin{gathered}
\sigma(0, t)\left[L\left(L^{\prime}\right)^{2}+\varepsilon L^{\prime}\right]=[\sigma(0, t)+\varepsilon] L, \\
L(0)=0 .
\end{gathered}
$$

We now analyze (4.8), (4.9) in various limits.

(i) $\varepsilon \rightarrow 0$.

We first seek a solution of (4.8) and (4.9) of the form

$$
L(t, \varepsilon)=L_{0}(t)+\varepsilon L_{1}(t)+\cdots .
$$

After substituting this expansion in (4.8) and (4.9) and solving the problems for $L_{0}(t)$ and $L_{1}(t)$, we find

$$
L(t, \varepsilon)=t+\frac{\varepsilon}{2} \ln \left[\left(e^{t}-1\right) / t\right]+0\left(\varepsilon^{2}\right) .
$$

We note from (4.11) that $L(t, \varepsilon) \sim t(1+\varepsilon / 4)+O\left(\varepsilon^{2}\right)$ as $t \rightarrow 0$ and $L(t, \varepsilon) \sim$ $t(1+\varepsilon / 2)+O\left(\varepsilon^{2}\right)$ as $t \rightarrow \infty$. Thus for short and long times, $L(t, \varepsilon)$ is proportional to $t$, which is typical of stress dominated transport [2].

(ii) $\varepsilon$ fixed, $t \rightarrow \infty$.

The long time solution of (4.8) is given by

$$
L(t, \varepsilon) \sim(1+\varepsilon)^{1 / 2} t \text { as } t \rightarrow \infty .
$$

For small $\varepsilon, L(t) \sim(1+(\varepsilon / 2)) t+0\left(\varepsilon^{2}\right)$ which correctly matches the $t \rightarrow \infty$ limit of the small $\varepsilon$ solution (4.11).

(iii) $\varepsilon$ fixed, $t \rightarrow 0$.

The small time solution is given by

$$
L(t, \varepsilon)=\sqrt{2 \varepsilon t}\left[1+\frac{t}{4 \varepsilon}\left(1+\frac{\varepsilon}{2}\right)+O\left(t^{2}\right)\right] .
$$

Note that the leading $\sqrt{t}$ behavior matches with the short time solution previously obtained in $\S 2$. By contrast to the long time solution, the $\varepsilon \rightarrow 0$ limit of the short time solution (4.13) does not match the $t \rightarrow 0$ limit of the small $\varepsilon$ solution (4.11). The short time solution (4.13) suggests that there exists an $O(\varepsilon)$ initial layer above which $L(t)$ behaves like $t$. An inner solution of (4.8) and (4.9), which describes the transition from the $\sqrt{t}$ to the $t$ behavior, is given by

$$
\begin{gathered}
L(t, \varepsilon)=\left(2 \varepsilon t+t^{2}\right)^{1 / 2}+O\left(\varepsilon^{2}\right) \\
t=O(\varepsilon) .
\end{gathered}
$$

In Fig. 2, we compare the function (4.14) with the numerical solution of (4.8) and (4.9). 


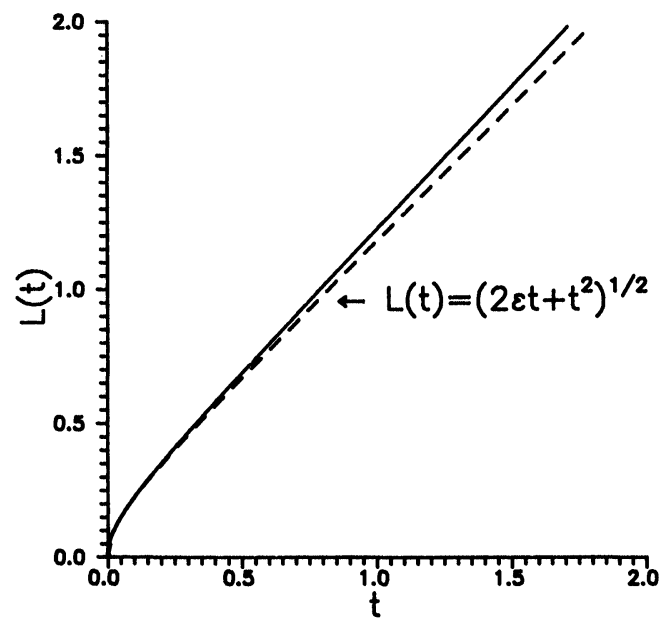

FIG. 2. The changing time history. The numerical solution of (4.8) and (4.9) (full line) is compared to the $t=0(\varepsilon)$ approximation (4.14) (dashed line) for $\varepsilon=0.2$.

In summary, we have assumed that the quasi-steady state approximation is a numerically valid approximation for small and moderate time. Using this approximation, we have shown that the undesirable " $\sqrt{t}$ " behavior is quickly followed by a " $t$ " behavior as $t \gg O(\varepsilon)$.

\section{REFERENCES}

[1] T. Alfrey, E. F. Gurnee, And W. G. Lloyd, Diffusion in glassy polymers, J. Polymer Sci. C, 12 (1966), pp. 249-261.

[2] R. W. Cox AND D. S. COHEN, A mathematical model for stress-driven diffusion in polymers, J. Polymer Sci. B: Polymer Physics, 27 (1989), pp. 589-602.

[3] D. S. COHEN AND A. B. White, Sharp fronts due to diffusion and stress at the glass transition in polymers, J. Polymer Sci. B: Physics Edition, 27 (1989), pp. 1731-1797.

[4] N. Thomas AND A. H. Windle, Transport of methanol in poly (methylmethocrylate), Polymer, 14 (1978), pp. 255-265.

[5] - A theory of Case II diffusion, Polymer, 23 (1982), pp. 529-542.

[6] D. S. COHEN AND T. ERNEUX, Free boundary problems in controlled release pharmaceuticals. I. Diffusion in glassy polymers, SIAM J. Appl. Math., 48 (1988), pp. 1451-1465.

[6b] - Free boundary problems in controlled release pharmaceuticals. II. Swelling-controlled release, SIAM J. Appl. Math., 48 (1988), pp. 1466-1474.

[7] D. S. Cohen, T. ERneux, AND M. J. WARd, Stress-driven controlled release, preprint, 1989.

[8] R. W. Cox, A model for stress-driven diffusion, Ph.D. thesis, California Institute of Technology, 1988.

[9] J. CRANK, The Mathematics of Diffusion, Second edition, Oxford University Press, Oxford, 1976. 